\title{
Replication of a genome-wide association study of panic disorder in a Japanese population
}

Takeshi Otowa ${ }^{1}$, Hisashi Tanii ${ }^{2}$, Nagisa Sugaya ${ }^{3}$, Eiji Yoshida ${ }^{3}$, Ken Inoue ${ }^{4}$, Shin Yasuda ${ }^{3}$, Takafumi Shimada ${ }^{1}$, Yoshiya Kawamura ${ }^{1}$, Mamoru Tochigi ${ }^{1}$, Takanobu Minato ${ }^{1}$, Tadashi Umekage ${ }^{1}$, Taku Miyagawa ${ }^{5}$, Nao Nishida ${ }^{5}$, Katsushi Tokunaga $^{5}$, Yuji Okazaki ${ }^{6}$, Hisanobu Kaiya ${ }^{3,7}$ and Tsukasa Sasaki ${ }^{8,9}$

Panic disorder (PD) is an anxiety disorder characterized by recurrent and unexpected panic attacks, subsequent worry and phobic avoidance. Although a number of association and linkage studies have been conducted, no gene has been identified as a susceptibility locus. We previously conducted a genome-wide association analysis of PD in 200 Japanese patients and the same number of controls, using a $500 \mathrm{~K}$ single nucleotide polymorphisms (SNPs) chip. In this study, we report a replication analysis of PD using the DigTag2 assay. The second stage sample consisted of 558 Japanese patients and 566 controls. Thirty-two markers were tested in a replication sample. As a result, no significant association was found after correction for multiple testing. However, the difference was observed at the nominal allele $P$-value $<0.05$ for two SNPs (rs6733840 and rs132617). We also conducted haplotype analyses of SNPs in the APOL3 and CLU genes. Our results failed to show any significant association with PD in these genes. Further studies on these variants with a larger sample size may be worth testing to confirm the results.

Journal of Human Genetics (2010) 55, 91-96; doi:10.1038/jhg.2009.127; published online 4 December 2009

Keywords: genome-wide association study (GWAS); Japanese population; panic disorder; replication; single nucleotide polymorphism (SNP)

\section{INTRODUCTION}

Panic disorder (PD) is an anxiety disorder characterized by recurrent and unexpected panic attacks, subsequent worry and phobic avoidance. Life prevalence of PD is $1-3 \%$ and twice as many women as men suffer from the disorder. ${ }^{1}$ The disorder frequently takes a chronic course, with many remissions and relapses. ${ }^{2}$ Genetic epidemiological studies including family and twin studies have shown that genetic as well as environmental factors have an important role in the pathogeneses of PD. First-degree relatives of proband with $\mathrm{PD}$ have an approximately fivefold increased risk of PD. ${ }^{3,4}$ Twin studies show that about $40 \%$ of the liability towards $\mathrm{PD}$ consists of heritable factors ${ }^{5-7}$ However, the etiology of $\mathrm{PD}$ is currently unknown.

Linkage analyses of PD have implicated several chromosomal regions including $1 \mathrm{q},{ }^{8} 2 \mathrm{q},{ }^{9} 7 \mathrm{p},{ }^{10,11} 9 \mathrm{q},{ }^{12} 12 \mathrm{q},{ }^{13} 13 \mathrm{q},{ }^{14,15} 15 \mathrm{q}^{9}$ and 22q. ${ }^{15}$ In association analyses, candidate genes including $H T R \quad 1 A,{ }^{16}$ $2 A,{ }^{17,18} C C K,{ }^{19}$ ADORA $2 A,{ }^{20} M A O A^{21}$ and $C O M T^{22,23}$ have been investigated. Most of the association studies were, however, of small sample size $(N<200)$ and the results were controversial. PD is considered to have polygenic- or oligogenic-multifactorial etiology, and most studies to date may be underpowered to detect the modest effect size of susceptibility loci.

We previously conducted a genome-wide association study of PD in the Japanese population using a $500 \mathrm{~K}$ single nucleotide polymorphism (SNP) chip. ${ }^{24}$ In the first stage, 200 patients and 200 controls were studied using the $500 \mathrm{~K}$ SNP chip (the GeneChip Human Mapping $500 \mathrm{~K}$ Array Set). A portion of the results of the first stage was previously reported. ${ }^{26}$ In the second stage, replication analysis of the SNPs, which were suggested in a previous study, was conducted in a Japanese second sample of PD (558 patients and 566 controls) using the DigiTag2 assay.

\section{MATERIALS AND METHODS}

Subjects

All patients and control subjects in the first and second stages were ethnically Japanese and were recruited in the vicinity of Tokyo and Nagoya, Japan. Subjects in the first stage consisted of 200 PD patients (64 males and 136 females; age $=39.5 \pm 9.4$ years, mean \pm s.d.) and 200 controls ( 64 males and 136 females; $38.2 \pm 9.3$ years). Details were described in a previous paper. ${ }^{24}$ Subjects for the second sample comprised 558 unrelated PD patients (175 males and 383

\footnotetext{
${ }^{1}$ Department of Neuropsychiatry, Graduate School of Medicine, the University of Tokyo, Tokyo, Japan; ${ }^{2}$ Department of Neuropsychiatry, Graduate School of Medicine, Mie University, Mie, Japan; ${ }^{3}$ Outpatient Clinic for Anxiety Disorders, Akasaka Mental Clinic, Tokyo, Japan; ${ }^{4}$ Department of Public Health, Fujita Health University School of Medicine, Aichi, Japan; ${ }^{5}$ Department of Human Genetics, Graduate School of Medicine, the University of Tokyo, Tokyo, Japan; ${ }^{6}$ Department of Neurology, Tokyo Metropolitan Matsuzawa Hospital, Tokyo, Japan; ${ }^{7}$ Research Center for Panic Disorder, Nagoya Mental Clinic, Nagoya, Japan; ${ }^{8}$ Health Service Center, the University of Tokyo, Tokyo, Japan and ${ }^{9}$ Office for Mental Health Support and Graduate School of Education, the University of Tokyo, Tokyo, Japan

Correspondence: Dr T Otowa, Department of Neuropsychiatry, Graduate School of Medicine, University of Tokyo 7-3-1 Hongo, Bunkyo, Tokyo 113-8655, Japan.

E-mail: totowa-psy@umin.ac.jp
}

Received 24 October 2009; accepted 6 November 2009; published online 4 December 2009 
females; age $=38.9 \pm 11.1$ years, 252 from Tokyo and 306 from Nagoya) and 566 controls ( 272 males and 294 females; age $=35.7 \pm 12.9$ years), who were recruited around Tokyo $(n=466)$ and the Nagoya-Tsu area $(n=100)$. For the multimarker haplotype analyses in the most susceptible genes in replication, subjects comprised 563 PD patients (177 males and 386 females; age $=38.5 \pm 10.8$ years; second sample and other five PD patients) and 506 controls ( 246 males and 260 females; age $=35.2 \pm 13.1$ years; second sample). Most of the sample overlapped with the second sample. The diagnosis was confirmed according to the DSM-IV criteria, ${ }^{25}$ using the $\mathrm{MINI}^{26}$ and clinical records. The controls received a short interview by one of the authors and filled out questionnaires to exclude the history of major psychiatric illness. Objective of this study was clearly explained and written informed consent was obtained from all subjects. The study was approved by the Ethical Committee of the Graduate School of Medicine, University of Tokyo.

\section{Genome-wide association analysis of PD}

In the first stage, 200 Japanese PD cases and the same number of controls were genotyped using the GeneChip Human Mapping $500 \mathrm{~K}$ Array Set (Affymetrix, Santa Clara, CA, USA) below for the replication in the second stage.

\section{SNP selection}

In the first stage, we employed the following quality control criteria as in Miyagawa et al. ${ }^{27}$ : call rate $\geqslant 95 \%$ in patients or controls, Hardy-Weinberg equilibrium (HWE) $P$-value $\geqslant 0.1 \%$ in controls and minor allele frequency $\geqslant 5 \%$ in all samples. As a result, we observed 48 SNPs on autosomal chromosomes and one SNP on X-chromosome in female subjects at the level of $P$-value $<10^{-4}$. For the second stage, 32 SNPs were selected out of 49 as follows. Among the 49 SNPs, 29 had a minor allele frequency of $\geqslant 10 \%$ in all subjects. Out of the 29 , rs 12227600 was excluded because it was located in the same linkage disequilibrium (LD) block with another of the 28 SNPs. Rs16985077 on X-chromosome was also excluded because the sample size was relatively limited and the analysis by sex may be underpowered. Thus, 27 SNPs were selected for the replication. As the DigiTag2 system simultaneously genotypes 32 SNP on one plate, five others (rs3804375, rs12579350, rs941184, rs3816995 and rs1383290) with the lowest $P$-values in the rest of the 49 SNPs (minor allele frequency $\geqslant 5 \%$; $P$-value $<10^{-4}$ ) were also selected and studied in the replication.

\section{Genotyping}

Genomic DNA was extracted from leukocytes by using the standard phenolchloroform method. Genotyping was conducted at the SNP-Typing Center (University of Tokyo) using the DigTag2 assay (Olympus, Tokyo, Japan) following the manufacturer's protocol. A multiplex SNP-typing method designated DigTag2 is suitable for genotyping an intermediate number of SNPs (tens to hundreds of sites) with a high conversion rate ( $>90 \%)$, high accuracy and low cost. ${ }^{28,29}$ The conversion rate is defined as the proportion of successfully genotyped SNPs among the total number of SNPs examined. The reproducibility of this assay was examined by duplicate experiments, resulting $100 \%$ identical between duplicate experiments.

\section{Haplotype analyses}

We conducted additional haplotype-based analyses to augment the signals of the most associated SNPs in known genes. We examined five SNPs in CLU (APOJ) and four in APOL3 (Figure 1). Besides rs17466684 and rs132617 investigated in the second stage, four tag SNPs (rs2279591, rs9331949, rs2279590 and rs9331888) in $C L U(A P O J)$ and three tag SNPs (rs3827346, rs1807672, rs132660) in APOL3 were selected from the HapMap database (www.hapmap. org). These tag SNPs were selected to include at least one SNP within each LD block, which was determined with the criteria $\mathrm{D}^{\prime} \geqslant 0.75$ using SNPbrowser software ver.3.5 (Applied Biosystems, Foster city, CA, USA). All SNPs were genotyped using the ABI PRISM 7900HT Sequence Detection System (Applied Biosystems), according to the manufacturer's protocol (Applied Biosystems),
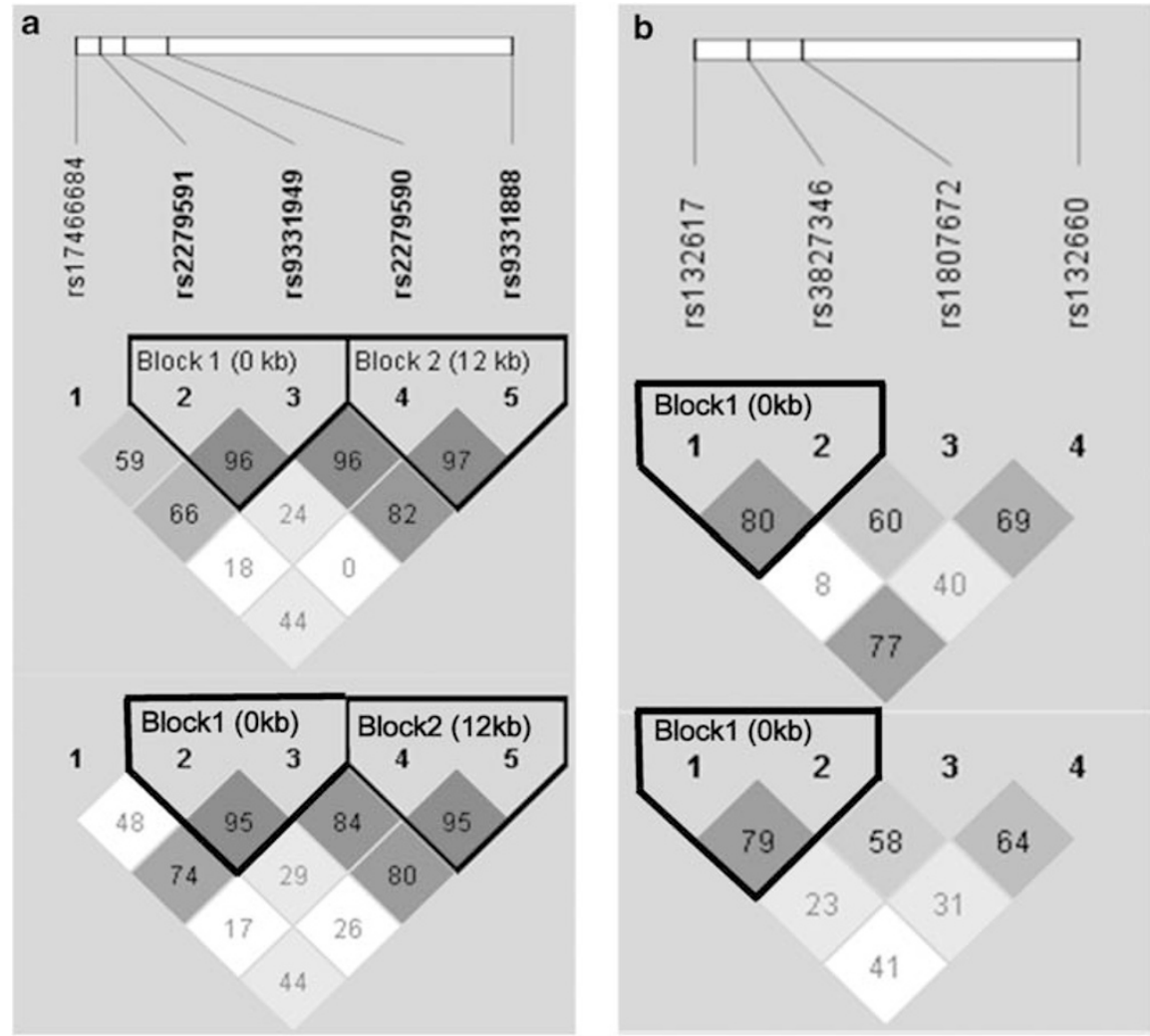

Figure 1 The strength of linkage disequilibrium (LD) between pairs of polymorphisms in patients and controls. The values of $D^{\prime}$ for patients are shown in the upper portion and those for controls in the lower portion. The heavy-line frames show suggested haplotype blocks. (a) CLU (or APOJ) (b) APOL3. 
with ABI PRISM 7900 SDS2 Software (Applied Biosystems). To check for typing errors, we re-genotyped rs9331949 in duplicated samples. The concordance rates between the genotypes using the TaqMan were $100 \%$.

\section{Data analyses}

Deviation from the HWE was tested using the $\chi^{2}$-test. Allele and genotype frequencies of the patients and control subjects were compared using the $\chi^{2}$-test. Significance for the result was set at $P<0.05$. Data analyses for replication and the TaqMan study were performed using the PLINK software package $^{30}$ (http://pngu.mgh.harvard.edu/purcell/plink/). LD and haplotype were analyzed in patients and controls who were studied using TaqMan method for all nine SNPs. $D^{\prime}$ was used to analyze pairwise LD. ${ }^{31}$ Haplotype block analysis was conducted using the Gabriel method. ${ }^{32}$ The HaploView 4.1 program was used to conduct the LD and haplotype block analyses. ${ }^{33}$ A power calculation was performed using the method as described elsewhere. ${ }^{34,35}$

\section{RESULTS}

\section{Replication analysis}

Three of 32 SNPs (SNP29, SNP30 and SNP31) were not successfully genotyped because of strong false-positive signals, which resulted from misligation in the encoding step. Therefore, 29 SNPs were investigated for the replication. Genotyping call rate was $99.57 \%$ in the 29 SNPs. Allele and genotype frequencies are summarized in Table 1. The genotype distributions of two SNPs were significantly deviated from the HWE (SNP16; $P=0.035$ in patients, SNP20; $P=0.024$ in controls, respectively). Other SNPs were within HWE $(P>0.05)$ in both patients and controls. Significant differences were observed in the allele and genotype frequency of the SNP2 (rs860554; allele $P=0.015$, genotype $P=0.043$ ), SNP4 (rs6733840; allele $P=0.021$ ) and SNP32 (rs132617; allele $P=0.025$ ) between patients and controls (Tables 1 and 2), although the associations did not reach statistical significance after Bonferroni correction. Risk alleles of SNP4 and SNP32 were the same as the first sample whereas risk allele of SNP2 was different from the first sample. SNP32 (rs132617) is located within $3 \mathrm{~kb}$ of the APOL3 gene and SNP4 (rs6733840) is located in an unknown gene. Four SNPs (SNP8, SNP12, SNP16 and SNP25) showed allele $P$-value between 0.05 and 0.1. Risk alleles of three SNPs (SNP12, SNP16 and SNP25) were the same as in the first sample, whereas risk allele of SNP8 was different from the first sample. SNP12 (rs17466684) is located within $1 \mathrm{~kb}$ of the CLU (or APOJ) gene and SNP25 (rs8013992) is located in the STON2 gene, whereas SNP16 (rs12420498) is not located in or adjacent to any gene.

\section{Haplotype analyses}

Among seven SNPs at the allele $P$-value of $<0.1$ in the second sample, risk alleles of five SNPs were the same as in the first sample. Among five SNPs, three SNPs are located in known genes including APOL3,

Table 1 Allele and genotype frequencies of 29 SNPs successfully genotyped in the second stage between patients and controls

\begin{tabular}{|c|c|c|c|c|c|c|c|c|}
\hline \multirow[b]{2}{*}{$S N P$} & \multirow[b]{2}{*}{ dbSNP (Major/minor) } & \multirow[b]{2}{*}{ Chromosome } & \multicolumn{2}{|c|}{ Minor allele frequency } & \multirow[b]{2}{*}{ P-value } & \multicolumn{2}{|c|}{ Genotype frequency ${ }^{\mathrm{a}}$} & \multirow[b]{2}{*}{ P-value } \\
\hline & & & Patients & Controls & & Patients & Controls & \\
\hline 1 & rs2806561(A/G) & $1 p 36$ & 0.49 & 0.48 & NS & $0.25 / 0.51 / 0.24$ & $0.25 / 0.54 / 0.21$ & NS \\
\hline 2 & rs860554 (C/T) & $1 q 32$ & 0.14 & 0.17 & 0.015 & $0.75 / 0.24 / 0.01$ & 0.69/0.28/0.03 & 0.043 \\
\hline 3 & rs6587428 (C/T) & $1 q 44$ & 0.41 & 0.43 & NS & $0.35 / 0.49 / 0.16$ & 0.33/0.49/0.18 & NS \\
\hline 4 & rs6733840 (T/C) & $2 q 14$ & 0.18 & 0.22 & 0.021 & 0.67/0.30/0.03 & 0.60/0.35/0.04 & 0.059 \\
\hline 5 & rs751056 (A/G) & $3 p 26$ & 0.37 & 0.34 & NS & $0.40 / 0.46 / 0.14$ & $0.44 / 0.45 / 0.12$ & NS \\
\hline 6 & rs3749380 (C/T) & $3 p 26$ & 0.34 & 0.36 & NS & $0.40 / 0.46 / 0.14$ & $0.41 / 0.46 / 0.13$ & NS \\
\hline 7 & rs3804375 (G/A) & $4 q 23$ & 0.08 & 0.07 & NS & $0.85 / 0.15 / 0.01$ & $0.87 / 0.13 / 0.00$ & NS \\
\hline 8 & rs4702982 (C/T) & $5 q 21$ & 0.23 & 0.26 & 0.097 & $0.58 / 0.37 / 0.05$ & 0.55/0.37/0.08 & NS \\
\hline 9 & rs2295767 (C/T) & $6 p 25$ & 0.15 & 0.15 & NS & $0.73 / 0.24 / 0.03$ & $0.72 / 0.25 / 0.03$ & NS \\
\hline 10 & rs2103868 (A/G) & $6 \mathrm{p} 21$ & 0.21 & 0.20 & NS & 0.63/0.32/0.05 & 0.63/0.33/0.04 & NS \\
\hline 11 & rs9372078 (A/T) & $6 q 25$ & 0.28 & 0.29 & NS & 0.52/0.39/0.09 & $0.51 / 0.41 / 0.08$ & NS \\
\hline 12 & rs17466684 (G/A) & $8 p 21$ & 0.20 & 0.17 & 0.061 & 0.65/0.30/0.05 & $0.69 / 0.28 / 0.03$ & NS \\
\hline 13 & rs2016795 (G/A) & $8 q 12$ & 0.14 & 0.12 & NS & $0.74 / 0.24 / 0.02$ & $0.77 / 0.22 / 0.01$ & NS \\
\hline 14 & rs2118261(A/G) & $8 q 12$ & 0.14 & 0.12 & NS & $0.74 / 0.24 / 0.02$ & $0.77 / 0.21 / 0.02$ & NS \\
\hline 15 & rs7070456 (C/T) & $10 p 12$ & 0.30 & 0.32 & NS & 0.48/0.43/0.09 & 0.45/0.46/0.9 & NS \\
\hline 16 & rs12420498 (T/A) & $11 q 23$ & 0.44 & 0.40 & 0.074 & 0.29/0.54/0.17 & $0.35 / 0.49 / 0.16$ & 0.080 \\
\hline 17 & rs12579350 (G/A) & $12 \mathrm{p} 13$ & 0.13 & 0.12 & NS & $0.76 / 0.22 / 0.02$ & 0.78/0.20/0.02 & NS \\
\hline 18 & rs11180828 (A/G) & $12 q 12$ & 0.18 & 0.18 & NS & 0.67/0.29/0.03 & 0.67/0.30/0.02 & NS \\
\hline 19 & rs2731006 (C/T) & $12 q 12$ & 0.34 & 0.36 & NS & $0.44 / 0.44 / 0.12$ & $0.43 / 0.41 / 0.16$ & NS \\
\hline 20 & rs2731000 (C/T) & $12 q 12$ & 0.34 & 0.36 & NS & $0.44 / 0.44 / 0.12$ & $0.44 / 0.41 / 0.15$ & NS \\
\hline 21 & rs941184 (C/T) & $12 q 13$ & 0.11 & 0.12 & NS & 0.79/0.20/0.01 & 0.77/0.22/0.01 & NS \\
\hline 22 & rs7299940 (C/G) & $12 q 24$ & 0.31 & 0.30 & NS & 0.46/0.46/0.08 & 0.48/0.43/0.08 & NS \\
\hline 23 & rs9302001 (C/T) & $13 q 32$ & 0.36 & 0.36 & NS & $0.42 / 0.43 / 0.14$ & $0.42 / 0.43 / 0.15$ & NS \\
\hline 24 & rs10872898 (G/C) & $14 q 11$ & 0.11 & 0.10 & NS & 0.80/0.19/0.01 & $0.81 / 0.18 / 0.01$ & NS \\
\hline 25 & rs8013992 (G/A) & $14 q 31$ & 0.14 & 0.12 & 0.095 & $0.74 / 0.23 / 0.03$ & $0.78 / 0.21 / 0.01$ & NS \\
\hline 26 & rs3816995 (G/A) & $17 q 25$ & 0.16 & 0.15 & NS & 0.70/0.28/0.02 & $0.73 / 0.24 / 0.03$ & NS \\
\hline 27 & rs1383290 (C/A) & $18 q 12$ & 0.11 & 0.11 & NS & 0.80/0.19/0.01 & 0.80/0.19/0.01 & NS \\
\hline 28 & rs11660890 (T/C) & $18 q 23$ & 0.47 & 0.48 & NS & $0.26 / 0.53 / 0.21$ & $0.28 / 0.48 / 0.24$ & NS \\
\hline 32 & rs132617 (C/T) & $22 q 12.3$ & 0.25 & 0.29 & 0.025 & 0.56/0.37/0.07 & $0.50 / 0.42 / 0.08$ & 0.076 \\
\hline
\end{tabular}

Abbreviation: NS, not significant

Three SNPs (SNP29, SNP30 and SNP31) were excluded for analyses because of conversion failure.

aDescribed as major homo/hetero/minor homo. 
Table 2 Allele frequencies in the first and second samples of SNPs showing allele $P<0.001$ in the first sample and $P<0.1$ in the second sample

\begin{tabular}{|c|c|c|c|c|c|c|c|c|c|}
\hline SNP & dbSNP (rs no.) & Chromosome & \multicolumn{2}{|c|}{$m A F$} & P-value & \multicolumn{2}{|c|}{$m A F$} & P-value & Gene \\
\hline $2^{\mathrm{a}}$ & rs860554 & $1 q 32$ & 0.17 & 0.05 & $4.68 \times 10^{-8}$ & 0.14 & 0.17 & 0.015 & $P K P 1$ \\
\hline 4 & rs6733840 & $2 q 14$ & 0.13 & 0.24 & $6.02 \times 10^{-5}$ & 0.18 & 0.22 & 0.021 & Unknown \\
\hline 16 & rs12420498 & $11 q 23$ & 0.47 & 0.33 & $5.20 \times 10^{-5}$ & 0.44 & 0.40 & 0.074 & - \\
\hline 25 & rs8013992 & $14 q 31$ & 0.18 & 0.06 & $1.45 \times 10^{-5}$ & 0.14 & 0.12 & 0.095 & STON2 \\
\hline 32 & rs132617 & $22 q 12.3$ & 0.21 & 0.34 & $7.16 \times 10^{-5}$ & 0.25 & 0.29 & 0.025 & APOL3 \\
\hline
\end{tabular}

Abbreviation: $\mathrm{mAF}$, minor allele frequency.

aRisk alleles of SNP2 and SNP8 were different between the first and second samples.

Table 3 Allele and genotype frequencies of SNPs in the CLU and APOL3 genes

\begin{tabular}{|c|c|c|c|c|c|c|c|}
\hline \multirow[b]{2}{*}{ Locus (Chr) } & \multirow[b]{2}{*}{$d b S N P$ (Major/Minor) } & \multicolumn{2}{|c|}{ Minor allele frequency } & \multirow[b]{2}{*}{ P-value } & \multicolumn{2}{|c|}{ Genotype frequency ${ }^{\mathrm{a}}$} & \multirow[b]{2}{*}{$P$-value } \\
\hline & & Patients & Controls & & Patients & Controls & \\
\hline \multirow[t]{3}{*}{$(8 \mathrm{p} 21)$} & rs2279591 (T/C) & 0.25 & 0.26 & 0.96 & $0.56 / 0.37 / 0.07$ & $0.56 / 0.37 / 0.07$ & 0.96 \\
\hline & rs9331949 (T/C) & 0.21 & 0.21 & 0.82 & $0.63 / 0.33 / 0.05$ & $0.60 / 0.37 / 0.03$ & 0.21 \\
\hline & rs2279590 (C/T) & 0.27 & 0.28 & 0.60 & $0.54 / 0.39 / 0.08$ & 0.53/0.39/0.09 & 0.84 \\
\hline APOL3 & rs132617 (C/T) & 0.25 & 0.29 & 0.03 & $0.56 / 0.38 / 0.06$ & $0.50 / 0.41 / 0.09$ & 0.10 \\
\hline \multirow[t]{3}{*}{ (22p12.3) } & rs3827346 (G/A) & 0.34 & 0.37 & 0.19 & $0.42 / 0.47 / 0.11$ & $0.39 / 0.48 / 0.13$ & 0.42 \\
\hline & rs1807672 (G/T) & 0.17 & 0.16 & 0.81 & $0.70 / 0.28 / 0.03$ & $0.70 / 0.28 / 0.02$ & 0.88 \\
\hline & rs132660 (T/G) & 0.13 & 0.13 & 0.76 & $0.75 / 0.24 / 0.01$ & $0.76 / 0.23 / 0.01$ & 0.83 \\
\hline
\end{tabular}

aDescribed as major homo/hetero/minor homo.

CLU (or APOJ) and STON2. We selected APOL3 and CLU (or APOJ) in which SNPs showed significant or marginally significant allele $P$-values. Allele and genotype frequencies of SNPs in the APOL3 and $C L U$ genes between patients and controls were shown in Table 3. The genotype distributions of rs9331949 in controls were nominally deviated from the HWE $(P=0.045)$. Other SNPs were within HWE $(P>0.05)$ in both patients and controls. Significant differences were observed in allele frequencies of rs17466684 and rs132617 $(P=0.019$ and 0.033 , respectively), although the statistical level became insignificant after correction for multiple testing. No significant difference was observed in allele frequencies of other SNPs or genotype frequencies of all SNPs between patients and controls.

The strength of LD denoted $\mathrm{D}^{\prime}$ between pairs of polymorphisms is shown in Figure 1. The LD pattern was broadly similar in the patient and control groups. In haplotype block analysis, two blocks consisting of SNP2-SNP3 (rs2279591-rs9331949) and SNP4-SNP5 (rs2279590rs9331888) were suggested in CLU; and a block consisting of SNP1SNP2 (rs132617-rs3827346) in APOL3 was suggested to be in strong $\mathrm{LD}\left(\mathrm{D}^{\prime} \geqslant 0.75\right)$. We then compared frequencies of the suggested three haplotypes between patients and controls in addition to two haplotypes consisting of all polymorphisms in each gene. A significant difference was observed in the frequency of the haplotype consisting of all polymorphisms between patients and controls in CLU (ATTCG: $P=0.035$; Table 4), whereas the statistical level became insignificant after correction for multiple testing. No significant difference was observed in frequencies of other haplotypes between both patients and controls (Table 4).

\section{DISCUSSION}

We conducted a two-stage association analysis of PD in the Japanese population. Genome-wide association analysis was conducted in the first stage and 32 candidate SNPs according to the first stage result were tested in the second stage using the DigiTag2 assay. Among 32 SNPs selected for replication, 29 SNPs were successfully genotyped. Although we did not find a significant association after Bonferroni correction, two SNPs (rs6733840 and rs132617) were associated with $\mathrm{PD}$ at $5 \%$ level with the same risk allele as in the first sample. We also conducted haplotype analyses of SNPs in the APOL3 and CLU genes. Our results failed to show any significant association with PD in either a single SNP or haplotypes in these genes. However, rs132617 in the APOL3 gene and rs17466684 in the CLU gene were associated with $\mathrm{PD}$ at $5 \%$ level. The haplotype ATTCG in the CLU gene was nominally associated with PD.

APOL proteins might be involved in lipid transport and metabolism. Six APOL genes (APOL1-6) are located near each other on the 22q12 locus. The cholesterol content of intracellular membranes of neurons might be involved in modulating gene transcription and signal transduction in the brain during neurodevelopment and in the 
Table 4 Haplotype frequencies according to haplotypes in each blocks and all polymorphisms of the CLU and APOL3 genes

\begin{tabular}{|c|c|c|c|c|}
\hline & \multirow[b]{2}{*}{ Haplotype } & \multicolumn{2}{|c|}{ Frequencies $^{\mathrm{a}}$} & \multirow[b]{2}{*}{ P-value } \\
\hline & & Patients & Controls & \\
\hline \multicolumn{5}{|l|}{$C L U$} \\
\hline \multicolumn{5}{|c|}{ SNP2-SNP3 } \\
\hline 1 & TT & 0.54 & 0.53 & 0.77 \\
\hline 2 & CT & 0.25 & 0.25 & 0.84 \\
\hline 3 & TC & 0.21 & 0.21 & 0.88 \\
\hline \multicolumn{5}{|c|}{ SNP4-SNP5 } \\
\hline 1 & CG & 0.43 & 0.40 & 0.14 \\
\hline 2 & $\mathrm{CC}$ & 0.30 & 0.32 & 0.28 \\
\hline 3 & $\mathrm{TC}$ & 0.27 & 0.28 & 0.63 \\
\hline \multicolumn{5}{|c|}{ SNP1-SNP2-SNP3-SNP4-SNP5 } \\
\hline 1 & GTTCG & 0.20 & 0.22 & 0.26 \\
\hline 3 & GTCCC & 0.19 & 0.19 & 0.89 \\
\hline 3 & ATTCG & 0.13 & 0.10 & 0.04 \\
\hline 4 & GCTCC & 0.12 & 0.13 & 0.29 \\
\hline 5 & GTTTC & 0.11 & 0.12 & 0.64 \\
\hline 6 & GCTCG & 0.09 & 0.07 & 0.13 \\
\hline 7 & GTTCC & 0.07 & 0.08 & 0.41 \\
\hline \multicolumn{5}{|c|}{ APOL3 } \\
\hline \multicolumn{5}{|c|}{ SNP1-SNP2 } \\
\hline 1 & CG & 0.63 & 0.59 & 0.12 \\
\hline 2 & TA & 0.22 & 0.25 & 0.07 \\
\hline 3 & $\mathrm{CA}$ & 0.12 & 0.11 & 0.62 \\
\hline \multicolumn{5}{|c|}{ SNP1-SNP2-SNP3-SNP4 } \\
\hline 1 & CGGT & 0.58 & 0.55 & 0.13 \\
\hline 2 & TAGT & 0.17 & 0.19 & 0.23 \\
\hline 3 & TATT & 0.05 & 0.06 & 0.26 \\
\hline
\end{tabular}

aHaplotypes whose frequencies were estimated $>5 \%$ were described.

adult. ${ }^{36}$ A postmortem study using the candidate gene cDNA array showed the upregulations of APOL1, APOL2 and APOL4 in the prefrontal cortex of schizophrenic patients, ${ }^{37}$ whereas recent association study showed no association of this gene cluster with schizophrenia. ${ }^{38}$ In this study, SNP rs132617 was nominally associated with PD. In haplotype analysis, we observed a trend of decrease in the frequency of the haplotype TA (s132617-rs3827346) in PD patients $(P=0.07)$. The result suggested that this haplotype might be protective against $\mathrm{PD}$.

Furthermore, Clusterin, which is a protein encoded by the CLU (or $A P O J)$ gene located on chromosome $8 \mathrm{p} 21$, might function in membrane lipid recycling. ${ }^{39}$ It may also be involved with ongoing synapse turnover. ${ }^{40}$ APOJ might regulate the process of amyloid- $\beta$ deposition in the brain of Alzheimer's disease. ${ }^{41}$ Some studies suggested a correlation between cholesterol levels and the intensity and frequency of panic attacks reported by PD patients. ${ }^{42,43}$ In our analyses, trends of increase in the frequencies of the allele A (rs17466684) and the haplotype ATTCG (rs17466684-rs9331888) in PD patients were observed. The results suggested that these, SNPs and haplotypes, might be a risk factor of PD. Previous findings from animal studies suggested that Clusterin increases post-ischemic damage and may exert a negative effect on the structural integrity and functionality of hippocampal neurons. ${ }^{44}$

Several limitations may be noted in this study. First, sample size (558 patients and 566 controls) might not be adequate. The second sample has statistical power of $>0.80($ alpha $=0.05)$ for the detection of the role of the polymorphism with minor allele frequency of 0.1 , when the genotype relative risk is $>1.52$. A larger sample is requested for the detection of genes with a smaller effect than this. Second, proportions of the subjects from Tokyo and those from the Nagoya-Tsu area were not same between patients and controls. However, all subjects were ethnically Japanese and minor allele frequencies of significant SNPs were not different between the subjects from Tokyo and those from the Nagoya-Tsu area (data not shown).

In conclusion, we conducted a replication analysis of a genomewide association study of PD in the Japanese population. As a result, no significant association with PD was found after correction for multiple testing. However, the difference was observed at the nominal allele $P$-value $<0.05$ for two SNPs (rs6733840 and rs132617). We also conducted haplotype analyses of SNPs in the APOL3 and CLU genes. Our results failed to show any significant association with PD in these genes. Further studies on these variants with a larger sample size may be worth testing to confirm the results.

\section{ACKNOWLEDGEMENTS}

This study is supported by Grant-in-Aid for Scientific Research on Priority Areas 'Comprehensive Genomics' and 'Applied Genomics' (no. 17019029) from the Ministry of Education, Culture, Sports, Science and Technology of Japan. We are grateful to Ms Yoshimi Ishibashi, Ms Yuko Ogasawara and Ms Kayoko Kato for excellent technical assistance. Most important, we thank the individuals who have participated in and contributed to this study.

1 Eaton, W. W., Kessler, R. C., Wittchen, H. U. \& Magee, W. J. Panic and panic disorder in the United States. Am. J. Psychiatry 151, 413-420 (1994).

2 Weissman, M. M., Bland, R. C., Canino, G. J., Faravelli, C., Greenwald, S., Hwu, H. G. et al. The cross-national epidemiology of panic disorder. Arch. Gen. Psychiatry 54, 305-309 (1997).

3 Crowe, R. R., Noyes, R., Pauls, D. L. \& Slymen, D. A family study of panic disorder. Arch. Gen. Psychiatry 40, 1065-1069 (1983).

4 Goldstein, R. B., Wickramaratne, P. J., Horwath, E. \& Weissman, M. M. Familial aggregation and phenomenology of 'early'-onset (at or before age 20 years) panic disorder. Arch. Gen. Psychiatry 54, 271-278 (1997).

5 Hettema, J. M., Neale, M. C. \& Kendler, K. S. A review and meta-analysis of the genetic epidemiology of anxiety disorders. Am. J. Psychiatry 158, 1568-1578 (2001).

6 Kendler, K. S., Neale, M. C., Kessler, R. C., Heath, A. C. \& Eaves, L. J. Major depression and generalized anxiety disorder. Same genes, (partly) different environments? Arch. Gen. Psychiatry 49, 716-722 (1992).

7 Torgersen, S. Genetics of neurosis. The effects of sampling variation upon the twin concordance ratio. Br. J. Psychiatry 142, 126-132 (1983).

8 Gelernter, J., Bonvicini, K., Page, G., Woods, S. W., Goddard, A. W., Kruger, S. et al. Linkage genome scan for loci predisposing to panic disorder or agoraphobia. Am. J. Med. Genet. 105, 548-557 (2001).

9 Fyer, A. J., Hamilton, S. P., Durner, M., Haghighi, F., Heiman, G. A., Costa, R. et al. A third-pass genome scan in panic disorder: evidence for multiple susceptibility loci. Biol. Psychiatry 60, 388-401 (2006).

10 Knowles, J. A., Fyer, A. J., Vieland, V. J., Weissman, M. M., Hodge, S. E., Heiman, G. A. et al. Results of a genome-wide genetic screen for panic disorder. Am. J. Med. Genet. 81, 139-147 (1998).

11 Crowe, R. R., Goedken, R., Samuelson, S., Wilson, R., Nelson, J., Noyes, Jr. R. et al. Genomewide survey of panic disorder. Am. J. Med. Genet. 105, 105-109 (2001).

12 Thorgeirsson, T. E., Oskarsson, H., Desnica, N., Kostic, J. P., Stefansson, J. G., Kolbeinsson, $\mathrm{H}$. et al. Anxiety with panic disorder linked to chromosome $9 \mathrm{q}$ in Iceland. Am. J. Hum. Genet. 72, 1221-1230 (2003).

13 Smoller, J. W., Acierno, J. S. Jr., Rosenbaum, J. F., Biederman, J., Pollack, M. H., Meminger, S. et al. Targeted genome screen of panic disorder and anxiety disorder proneness using homology to murine QTL regions. Am. J. Med. Genet. 105, 195-206 (2001).

14 Weissman, M. M., Fyer, A. J., Haghighi, F., Heiman, G., Deng, Z., Hen, R. et al. Potential panic disorder syndrome: clinical and genetic linkage evidence. Am. J. Med. Genet. 96, 24-35 (2000) 
15 Hamilton, S. P., Fyer, A. J., Durner, M., Heiman, G. A., Baisre de Leon, A., Hodge, S. E. et al. Further genetic evidence for a panic disorder syndrome mapping to chromosome 13q. Proc. Natl. Acad. Sci. USA 100, 2550-2555 (2003).

16 Rothe, C., Gutknecht, L., Freitag, C., Tauber, R., Mossner, R., Franke, P. et al. Association of a functional $1019 \mathrm{C}>\mathrm{G}$ 5-HT1A receptor gene polymorphism with panic disorder with agoraphobia. Int. J. Neuropsychopharmacol. 7, 189-192 (2004).

17 Inada, Y., Yoneda, H., Koh, J., Sakai, J., Himei, A., Kinoshita, Y. et al. Positive association between panic disorder and polymorphism of the serotonin $2 \mathrm{~A}$ receptor gene. Psychiatry Res. 118, 25-31 (2003).

18 Rothe, C., Koszycki, D., Bradwejn, J., King, N., De Luca, V., Shaikh, S. et al. Association study of serotonin-2A receptor gene polymorphism and panic disorder in patients from Canada and Germany. Neurosci. Lett. 363, 276-279 (2004).

19 Ebihara, M., Ohba, H., Hattori, E., Yamada, K. \& Yoshikawa, T. Transcriptional activities of cholecystokinin promoter haplotypes and their relevance to panic disorder susceptibility. Am. J. Med. Genet. 118, 32-35 (2003).

20 Deckert, J., Nothen, M. M., Franke, P., Delmo, C., Fritze, J., Knapp, M. et al. Systematic mutation screening and association study of the A1 and $\mathrm{A} 2 \mathrm{a}$ adenosine receptor genes in panic disorder suggest a contribution of the A2a gene to the development of disease. Mol. Psychiatry 3, 81-85 (1998).

21 Deckert, J., Catalano, M., Syagailo, Y. V., Bosi, M., Okladnova, O., Di Bella, D. et al. Excess of high activity monoamine oxidase A gene promoter alleles in female patients with panic disorder. Hum. Mol. Genet. 8, 621-624 (1999).

22 Hamilton, S. P., Slager, S. L., Heiman, G. A., Deng, Z., Haghighi, F., Klein, D. F. et al. Evidence for a susceptibility locus for panic disorder near the catechol-0-methyltransferase gene on chromosome 22. Biol. Psychiatry 51, 591-601 (2002).

23 Woo, J. M., Yoon, K. S., Choi, Y. H., Oh, K. S., Lee, Y. S. \& Yu, B. H. The association between panic disorder and the L/L genotype of catechol-O-methyltransferase. J. Psychiatr. Res. 38, 365-370 (2004).

24 Otowa, T., Yoshida, E., Sugaya, N., Yasuda, S., Nishimura, Y., Inoue, K. et al. Genomewide association study of panic disorder in the Japanese population. J. Hum. Genet. 54, 122-126 (2009).

25 American Psychiatric Association. Diagnostic and Statistical Manual of Mental Disorders 4th ed. (American Psychiatric Association, Washington DC, 1994).

26 Sheehan, D. V., Lecrubier, Y., Sheehan, K. H., Amorim, P., Janavs, J., Weiller, E. et al. The Mini-International Neuropsychiatric Interview (MINI): the development and validation of a structured diagnostic psychiatric interview for DSM-IV and ICD-10. J. Clin. Psychiatry 59 (Suppl 20), 22-33; Quiz 34-57 (1998).

27 Miyagawa, T., Nishida, N., Ohashi, J., Kimura, R., Fujimoto, A., Kawashima, M. et al. Appropriate data cleaning methods for genome-wide association study. J. Hum. Genet. 53, 886-893 (2008)

28 Nishida, N., Tanabe, T., Hashido, K., Hirayasu, K., Takasu, M., Suyama, A. et al. DigiTag assay for multiplex single nucleotide polymorphism typing with high success rate. Anal. Biochem. 346, 281-288 (2005).
29 Nishida, N., Tanabe, T., Takasu, M., Suyama, A. \& Tokunaga, K. Further development of multiplex single nucleotide polymorphism typing method, the DigiTag2 assay. Anal. Biochem. 364, 78-85 (2007).

30 Purcell, S., Neal, B., Todd-Brown, K., Thomas, L., Ferreira, M. A., Bender, D. et al. PLINK: a tool set for whole-genome association and population-based linkage analyses. Am. J. Hum. Genet. 81, 559-575 (2007).

31 Lewontin, R. C. The Interaction of Selection and Linkage. I. General Considerations; Heterotic Models. Genetics 49, 49-67 (1964).

32 Gabriel, S. B., Schaffner, S. F., Nguyen, H., Moore, J. M., Roy, J., Blumenstiel, B. et al. The structure of haplotype blocks in the human genome. Science 296, 2225-2229 (2002).

33 Barret, J. C., Fry, B., Maller, J. \& Daly, M. J. Haploview: analysis and visualization of LD and haplotype maps. Bioinformatics 21, 263-265 (2005).

34 Ohashi, J., Yamamoto, S., Tsuchiya, N., Hatta, Y., Komata, T., Matsushita, M. et al. Comparison of statistical power between $2 * 2$ allele frequency and allele positivity tables in case-control studies of complex disease genes. Ann. Hum. Genet. 65, 197-206 (2001).

35 Ohashi, J. \& Tokunaga, K. The expected power of genome-wide linkage disequilibrium testing using single nucleotide polymorphism markers for detecting a low-frequency disease variant. Ann. Hum. Genet. 66, 297-306 (2002).

36 Vanhollebeke, B. \& Pays, E. The function of apolipoproteins L. Cell Mol. Life. Sci. 63 , 1937-1944 (2006).

37 Mimmack, M. L., Ryan, M., Baba, H., Navarro-Ruiz, J., Iritani, S., Faull, R. L. et al. Gene expression analysis in schizophrenia: reproducible up-regulation of several members of the apolipoprotein $L$ family located in a high-susceptibility locus for schizophrenia on chromosome 22. Proc. Natl. Acad. Sci. USA. 99, 4680-4685 (2002).

38 McGhee, K. A., Morris, D. W., Schwaiger, S., Nangle, J. M., Donohoe, G., Clarke, S. et al. Investigation of the apolipoprotein-L (APOL) gene family and schizophrenia using a novel DNA pooling strategy for public database SNPs. Schizophr. Res. 76, 231-238 (2005).

39 Jones, S. E. \& Jomary, C. Clusterin. Int. J. Biochem. Cell Biol. 34, 427-431 (2002).

40 Danik, M. Chabot, J. G. Hassan-Gonzalez, D., Suh, M. \& Quirion, R. Localization of sulfated glycoprotein-2/clusterin mRNA in the rat brain by in situ hybridization. J. Comp. Neurol. 334, 209-227 (1993).

41 DeMattos, R. B., Cirrito, J. R., Parsadanian, M., May, P. C., O'Dell, M. A., Taylor, J. W. et al. ApoE and clusterin cooperatively suppress Abeta levels and deposition: evidence that ApoE regulates extracellular Abeta metabolism in vivo. Neuron 41, 193-202 (2004).

42 Hayward, C., Taylor, C. B., Roth, W. T., King, R. I \& Agras, W. S. Plasma lipid levels in patients with panic disorder or agoraphobia. Am. J. Psychiatry 146, 917-919 (1989).

43 Agargun, M. Y., Kara, H., Algun, E., Sekeroglu, R. \& Trakcioglu, M. High cholesterol levels in patients with sleep panic. Biol. Psychiatry. 40, 1064-1065 (1996).

44 Hakkoum, D., Imhof, A., Vallet, P. G., Boze, H., Moulin, G., Charnay, Y. et al. Clusterin increases post-ischemic damages in organotypic hippocampal slice cultures. J. Neurochem. 106, 1791-1803 (2008). 